\title{
RAYLEIGH-BÉNARD CONVECTION WITH ROTATION AT SMALL PRANDTL NUMBERS
}

\author{
Guenter Ahlers and Kapil M. S. Bajaj \\ Department of Physics and Center for Nonlinear Science \\ University of California, Santa Barbara, California 93106, USA
}

\begin{abstract}
This paper reviews results from and future prospects for experimental studies of Rayleigh-Bénard convection with rotation about a vertical axis. At dimensionless rotation rates $0 \leq \Omega \leq 20$ and for Prandtl numbers $\sigma \simeq 1$, Küppers-Lortz-unstable patterns offered a unique opportunity to study spatio-temporal chaos immediately above a supercritical bifurcation where weakly-nonlinear theories in the form of Ginzburg-Landau (GL) or Swift-Hohenberg ( $\mathrm{SH}$ ) equations can be expected to be valid. However, the dependence of the time and length scales of the chaotic state on $\epsilon \equiv$ $\Delta T / \Delta T_{c}-1$ was found to be different from the expected dependence based on the structure of GL equations. For $\Omega \gtrsim 70$ and $0.7 \lesssim \sigma \lesssim 5$ patterns were found to be cellular near onset with local four-fold coordination. They differ from the theoretically expected Küppers-Lortz-unstable state. Stable as well as intermittent defect-free rotating square lattices exist in this parameter range.

Smaller Prandtl numbers $(0.16 \lesssim \sigma \lesssim 0.7$ ) can only be reached in mixtures of gases. These fluids are expected to offer rich future opportunities for the study of a line of tricritical bifurcations, of supercritical Hopf bifurcations to standing waves, of a line of codimension-two points, and of a codimension-three point.
\end{abstract}

\section{INTRODUCTION}

Convection in a thin horizontal layer of a fluid heated from below (Rayleigh-Bénard convection or RBC) has become a paradigm for the study of pattern formation. [1] It evolves from the spatially-uniform pure-conduction state via a supercritical bifurcation when the temperature difference $\Delta T$ is increased beyond a critical value $\Delta T_{c}$. It reveals numerous interesting phenomena including spatio-temporal chaos (STC) as $\epsilon \equiv \Delta T / \Delta T_{c}-1$ grows. [1] Many of these phenomena have been studied in detail recently [2-21], using primarily compressed gases as the fluid, sensitive shadowgraph flowvisualization, image analysis, and quantitative heat-flux measurements [16]. However, 
as $\Delta T_{c}$ is approached from above and $\epsilon$ becomes small enough for the pattern-formation problem to become theoretically tractable by weakly-nonlinear methods, the system becomes relatively simple and its behavior can be described in potential (or variational) form. Then the steady-state pattern is time independent. In the absence of perturbing boundaries it consists of parallel rolls as shown in Fig. 1a [17] and as predicted theoretically [22] already 33 years ago.
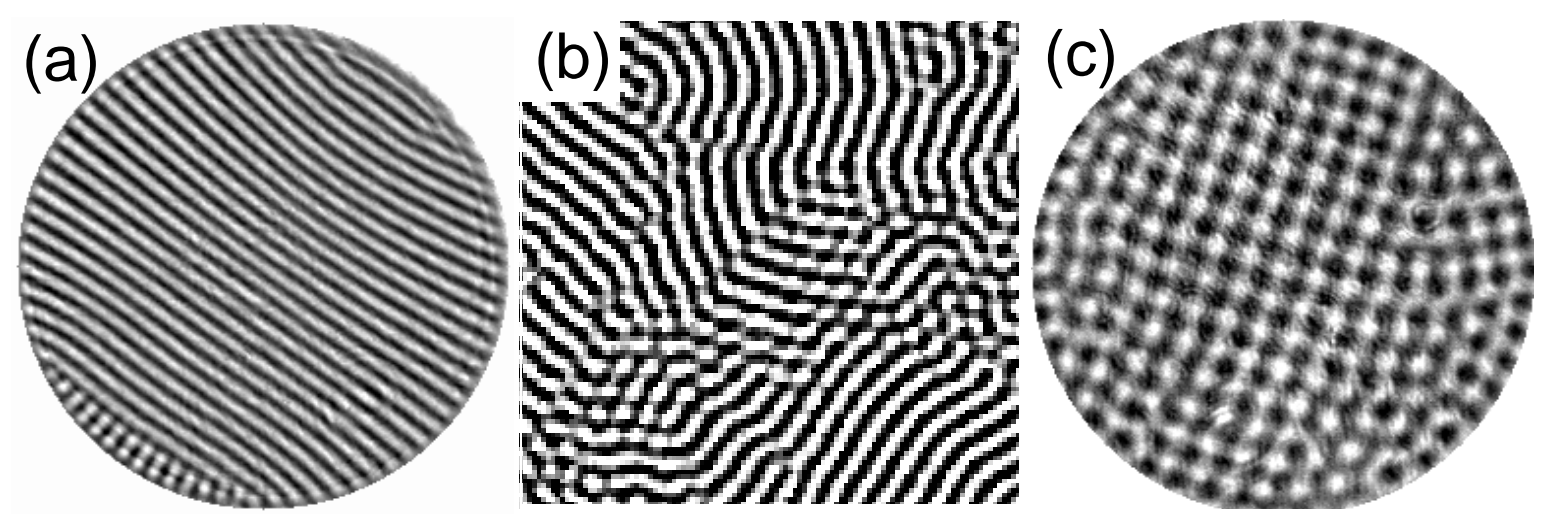

Fig. 1. Convection patterns for small $\epsilon$. (a) is for $\Omega=0$ and Ar gas with $\sigma=0.69$ and $\epsilon=0.07$ (from Ref. [17]). It shows the predicted [22] straight-roll pattern. (b) is for $\Omega=15.4$ and $\mathrm{CO}_{2}$ at a pressure of 32 bar with $\sigma=1.0$ and $\epsilon=0.05$ (from Ref. [23]). It is a typical pattern in the Küppers-Lortz-unstable range. (c) is for Argon at 40 bar with $\sigma=0.7, \Omega=145$, and $\epsilon=0.04$ (from Ref. [24]); it shows no evidence of the Küppers-Lortz instability, and instead consists of a slowly-rotating square lattice.

The system becomes much more complex and interesting even near onset when it is rotated about a vertical axis with an angular velocity $\vec{\Omega}$. In that case the coriolis force proportional to $\vec{\Omega} \times \vec{v}$ acts on the fluid (here $\vec{v}$ is the fluid velocity field in the rotating frame) and renders the system non-variational. Thus time dependent states can occur arbitrarily close to onset. Since the bifurcation remains supercritical for $\Omega>0$, the flow amplitudes still grow continuously from zero and the usual weakly-nonlinear theories, for instance in the form of GL or SH equations, should remain applicable. Thus one may expect interesting new effects to occur in a theoretically tractable parameter range.

Indeed it was predicted [25-27] that, for $\Omega>\Omega_{c}$, the primary bifurcation from the conduction state should be to parallel rolls which are unstable. Although $\Omega_{c}$ depends on the Prandtl number $\sigma$ (the ratio of the kinematic viscosity $\nu$ to the thermal diffusivity $\kappa$ ), it has a value near 14 for the $\sigma$-values near unity which are characteristic of compressed gases $(\Omega$ is made dimensionless by scaling time with the vertical viscous diffusion time $d^{2} / \nu$ where $d$ is the cell thickness). The instability is to plane-wave perturbations which are advanced relative to the rolls at an angle $\Theta_{K L}$ in the direction of $\vec{\Omega}$. This phenomenon is known as the Küppers-Lortz instability. A snapshot [23] of the resulting nonlinear state of convection is shown in Fig. 1b. The pattern consists of domains of rolls which 
incessantly replace each other, primarily by irregular domain-wall motion. [5,23,28-31] The spatial and temporal behavior suggests the term "domain chaos" for this state. We discuss this state in the next Section.

Theoretically, the KL instability is expected to persist near onset up to large values of $\Omega$. Thus it was a surprise that the patterns found in experiments near onset changed dramatically when $\Omega$ was increased. [24] For $\Omega \gtrsim 70$, there was no evidence of the characteristic domain chaos until $\epsilon$ was increased well above 0.1. At smaller $\epsilon$, slowly-rotating, aesthetically appealing, square lattices were encountered. Since these experimental observations are very new, it remains to be seen whether a reasonable explanation can be offered. They will be discussed in Sect. III.

Finally, we look forward to as yet unrealized experimental opportunities which this system has to offer in the parameter range of Prandtl numbers well below unity. Pure fluids (with rare exceptions [32]) have $\sigma \gtrsim 0.7$. Recently it was shown $[17,19]$ that smaller values of $\sigma$ can be reached by mixing two gases, one with a large and the other with a small atomic or molecular weight. The most extreme example readily available is a mixture of $\mathrm{H}_{2}$ and Xe. Prandtl numbers as small as 0.16 can be reached. In the range $\sigma \lesssim 0.6$, a number of interesting new phenomena are predicted to occur. [27,33] In the $\sigma-\Omega$ plane they include subcritical bifurcations below a line of tricritical bifurcations, [34] Hopf bifurcations to standing waves, a line of codimension-two points where the Hopf bifurcation meets the stationary bifurcation, and a codimension-three point where the codimension-two line and the tricritical line meet. The opportunities for research in this parameter range are outlined in Sect. IV.

\section{KÜPPERS-LORTZ DOMAIN-CHAOS}

For $\sigma \gtrsim 0.33$, the bifurcation to convection in the presence of rotation is expected to be supercritical both below and above $\Omega_{c}$. Thus the KL instability offers a rare opportunity to study STC in a system where the average flow amplitude evolves continuously from zero and where thus weakly-nonlinear theories might be expected to be applicable. After receiving only limited attention for several decades [25-29,35,36], the opportunity to study STC has led to a recent increase in activity both theoretically and experimentally $[16,23,37-43]$. Indeed, as predicted theoretically, [25] the straight rolls at the onset of convection for dimensionless rotation rates $\Omega>\Omega_{c}$ are unstable to another set of rolls oriented at an angle $\Theta_{K L}$ with respect to the original rolls along the direction of rotation. In the spatially extended system this leads to the co-existance of domains of rolls of more or less uniform orientation with other domains of a different orientation. A typical example is shown in Fig. 1b. Experiments by Heikes and Busse $[28,29]$ with water and using shadowgraph visualization rather far from onset $(\epsilon \gtrsim 0.5)$ established qualitatively the existence of the KL instability. The replacement of a given domain of rolls proceeded via domain-wall propagation. More recently the KL instability was investigated with shadowgraph flow-visualization very close to onset. It was demonstrated that the bifurcation is indeed supercritical, and that the instability leads to a 
continuous domain switching through a mechanism of domain-wall propagation also at small $\epsilon$. $[5,23,30,31]$ This qualitative feature has been reproduced by Tu and Cross [39] in numerical solutions of appropriate coupled GL equations, as well as by Neufeld et al. [41] and Cross et al. [42] through numerical integration of a generalized SH equation.
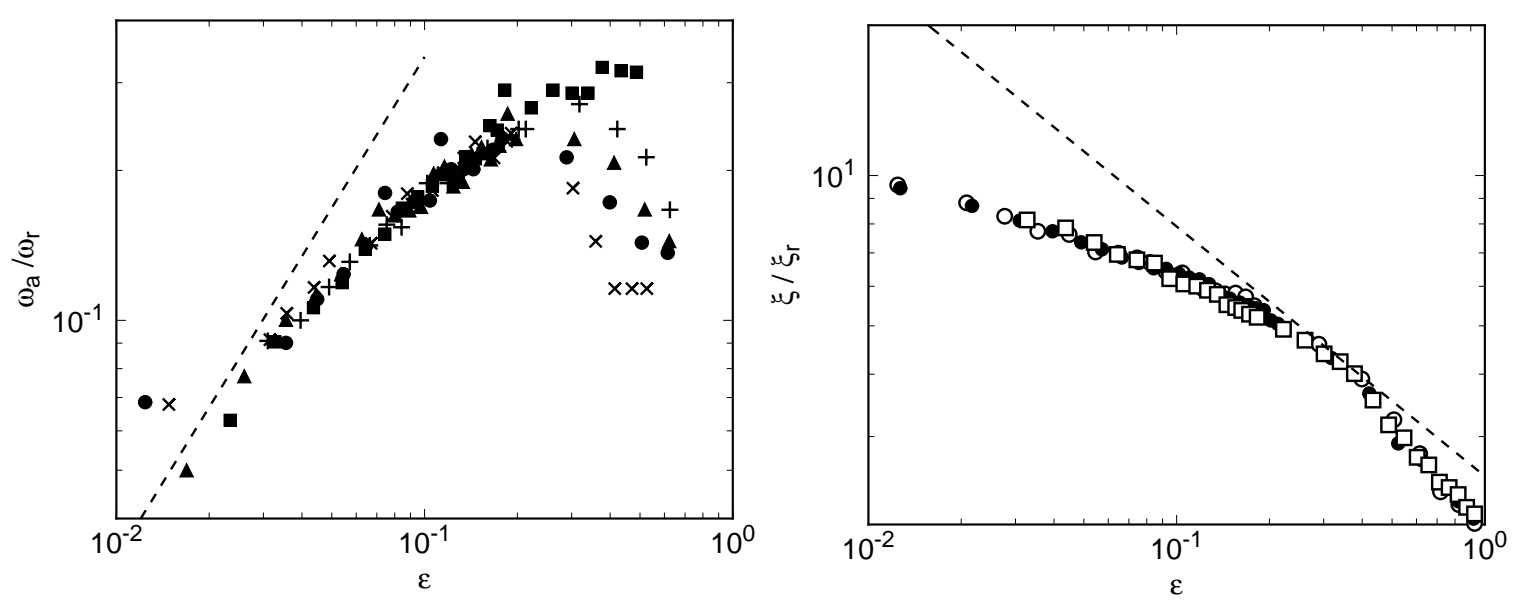

Fig. 2. The characteristic frequencies $\omega_{a}$ (left) and lengths $\xi$ (right) of the KL state. The data were divided by $\Omega$-dependent constants $\omega_{r}$ and $\xi_{r}$ so as to collapse them onto single curves. The dashed lines are shown for reference and have the slopes 1 for $\omega_{a}$ and $-1 / 2$ for $\xi$ which correspond to the theoretically expected exponents of the time and length scales near onset. The data sets cover approximately the range $14 \lesssim \Omega \lesssim 20$. See Refs. [23], [30], and [31] for details.

Of interest are the time and length scales of the KL instability near onset. The GL model assumes implicitly a characteristic time dependence which varies as $\epsilon^{-1}$ and a correlation length which varies as $\epsilon^{-1 / 2}$. We measured a correlation length given by the inverse width of the square of the modulus of the Fourier transform as well as a domainswitching frequency as revealed in Fourier space, and obtained the data in Fig. 2. [23,30] These results seem to be inconsistent with GL equations since they show that the time in the experiment scales approximately as $\epsilon^{-1 / 2}$ and that the two-point correlation length scales approximately as $\epsilon^{-1 / 4}$. These results also differ from numerical results based on a generalized SH equation [42] although the range of $\epsilon$ in the numerical work is rather limited. We regard the disagreement between experiment and theory as a major problem in our understanding of STC. [44] 


\section{SQUARE PATTERNS AT MODEST $\sigma$}

Motivated by the unexpected scaling of length and time with $\epsilon$ for the KL state at $\Omega \lesssim 20$, new investigations were undertaken recently in which the range of $\Omega$ was significantly extended to larger values. Contrary to theoretical predictions [27,33], it was found [24] in preliminary work that for $\Omega \gtrsim 70$ the nature of the pattern near onset changed qualitatively although the bifurcation remained supercritical. Square patterns like the one shown in Fig. 1c were stable, instead of typical KL patterns like the one in Fig. 1b. The squares occurred both when Argon with $\sigma=0.69$ was used and when the fluid was water with $\sigma \simeq 5$. The occurrence of squares in this system is completely unexpected and not predicted by theory; according to the theory the KL instability should continue to be found near onset also at these higher values of $\Omega$. Thus the preliminary work has uncovered a major disagreement with theoretical predictions in a parameter range where one might have expected the theory to be reliable. We believe that this calls for a systematic experimental study over appropriate parameter ranges. We expect to explore the range $0 \lesssim \Omega \lesssim 400$ and $0.7 \lesssim \sigma \lesssim 5$ in the near future, and hope that this will shed light on the extent and origin of the difference between the physical system and the predictions.

\section{THE RANGE $0.16<\sigma<0.7$}

When a RBC system is rotated about a vertical axis, the critical Rayleigh number $R_{c}(\Omega)$ increases as shown in Fig. 3a. $R_{c}(\Omega)$ is predicted to be independent of $\sigma$, and experiment [30] and theory [45] for it are in excellent agreement. For $\sigma>0.33$ the bifurcation is expected to be supercritical and to lead to KL chaos unless $\Omega$ is quite large. As discussed above in Sect. III, our recent preliminary experiments have cast doubt upon this; for $\Omega \gtrsim 70$ we found square patterns which are clearly unrelated to the typical KL domains. For large $\Omega$ and $\sigma<0.68$, the stationary bifurcation is predicted [33] to be preceded by a supercritical Hopf bifurcation; but for $\sigma>0.33$ we do not expect to reach values of $\Omega$ sufficiently high to encounter this in the experiment.

The range $0.16 \lesssim \sigma \lesssim 0.33$ is truly remarkable because of the richness of the bifurcation phenomena which occur there when the system is rotated. For instance, for $\sigma=0.26$ there is a range from $\Omega \simeq 16$ to 190 over which the bifurcation is predicted to be subcritical. This is shown by the dashed section of the curve in Fig. 3c. The subcritical range depends on $\sigma$. In Fig. 3b it covers the area below the dashed curve. Thus, the dashed curve is a line of tricritical bifurcations. [34] It has a maximum in the $\Omega-\sigma$ plane, terminating in a "tricritical endpoint". An analysis of the bifurcation phenomena which occur near it in terms of Landau equations may turn out to be interesting. One may expect path-renormalization [46] of the classical exponents in the vicinity of the maximum. We are not aware of equivalent phenomena in equilibrium phase transitions, although presumably they exist in as yet unexplored parameter ranges. 

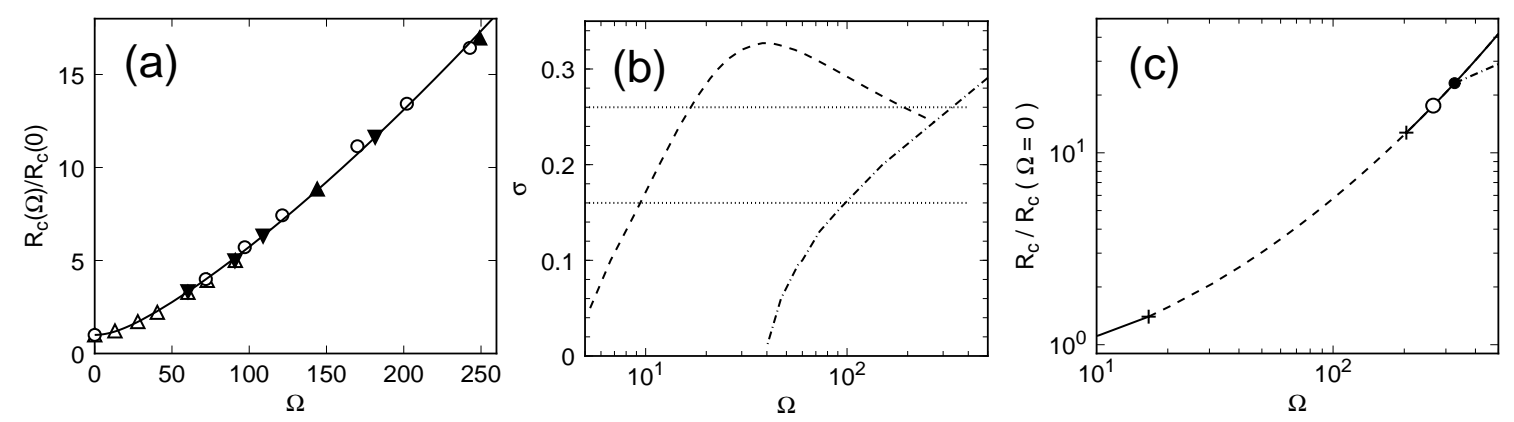

Fig. 3. The bifurcation diagram for RBC with rotation about a vertical axis. (a.) Experimental and theoretical results for $R_{c}(\Omega)$ obtained with water (open circles) and Ar at three different pressures (triangles) on linear scales. After Ref. [24]. This curve is expected to be independent of $\sigma$. (b.) The dashed curve gives the predicted tricritical line in the $\Omega-\sigma$ plane (the $\Omega$ axis is logarithmic). The dash-dotted line is the predicted codimension-two line where the Hopf bifurcation is expected to meet the stationary bifurcation [e.g. the solid circle for $\sigma=0.26$ in Fig. (c)]. For $\sigma=0.24$ the codimensiontwo line is expected to intersect the tricritical line, leading to the codimension-three point shown by the open circle in (c.). The upper dotted line in (b.) corresponds to the path represented in (c.). The lower dotted line in (b.) represents the lowest $\sigma$-value accessible to experiment using gas mixtures. (c.) The solid and dashed lines show the critical Rayleigh number as a function of $\Omega$ as in (a), but on logarithmic scales. The dashed line shows the range over which the bifurcation is predicted to be subcritical for $\sigma=0.26$. The two plusses, indicating the limits of this range, are the tricritical points for this $\sigma$-value. The dash-dotted line at large $\Omega$ shows the predicted Hopf bifurcation for $\sigma=0.26$.

At relatively large $\Omega$, the stationary bifurcation (regardless of whether it is superor sub-critical) is predicted to be preceded by a supercritical Hopf bifurcation which is expected to lead to standing waves of convection rolls. [33] Standing waves are relatively rare; usually a Hopf bifurcation in a spatially-extended system leads to traveling waves. An example is shown by the dash-dotted line near the right edge of Fig. 3b. As can be seen there, the Hopf bifurcation terminates at small $\Omega$ at a codimension-two point on the stationary bifurcation which, depending on $\sigma$, can be super- or sub-critical. The line of codimension-two points is shown in Fig. 3a as a dash-dotted line. One sees that the tricritical line and the codimension-two line meet at a codimension-three point, located at $\Omega \simeq 270$ and $\sigma \simeq 0.24$. We note that this is well within the parameter range accessible to our experiments. We are not aware of any experimentally-accessible examples of codimension-three points. This particular case should be accessible to analysis by weakly-nonlinear theories, and a theoretical description in terms of GL equations would be extremely interesting and could be compared with experimental measurements.

The $\sigma$-range of interest is readily accessible to us by using mixtures of a heavy and a light gas. [19] Values of $\sigma$ vs. the mole fraction $x$ of the heavy component for a typical pressure of 22 bar and at $25^{\circ} \mathrm{C}$ are shown in Fig. 4. An important question in this 
relation is whether the mixtures will behave in the same way as pure fluids with the same $\sigma$. We believe that to a good approximation this is the case because the Lewis numbers are of order one. This means that heat diffusion and mass diffusion occur on similar time scales. In that case, the concentration gradient will simply contribute to the buoyancy force in synchrony with the thermally-induced density gradient, and thus the critical Rayleigh number will be reduced. Scaling bifurcation lines by $R_{c}(\Psi)$ ( $\Psi$ is the separation ratio of the mixture) will mostly account for the mixture effect. To a limited extent we showed already that this is the case. $[17,19]$ In more recent work we have begun to show that the bifurcation line $R_{c}(\Omega) / R_{c}(0)$ is independent of $\Psi$. Nonetheless we recognize that a theoretical investigation of this issue will be very important.

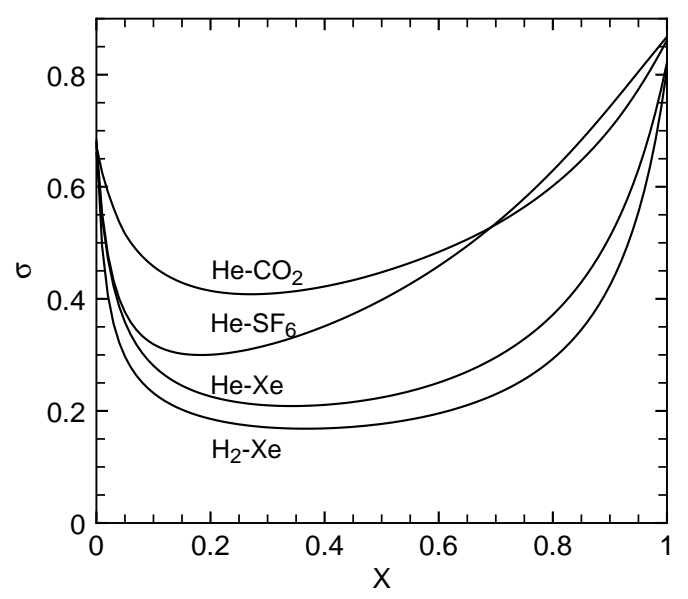

Fig. 4. The Prandtl number $\sigma$ as a function of the mole fraction $x$ of the heavy component for three gas mixtures at a pressure of 22 bar and at $25^{\circ} \mathrm{C}$. From Ref. [19].

Assuming that the mixtures behave approximately like pure fluids, we see that the codimension-three point can be reached using either $\mathrm{H}_{2}$-Xe or He-Xe mixtures. The tricritical point can be reached also using $\mathrm{He}_{-} \mathrm{SF}_{6}$.

\section{ACKNOWLEDGMENT}

The contents of this review is based on the work of many members of our groups, both at Santa Barbara and at Los Alamos. These include Robert Ecke, Yu-Chou Hu, Jun Liu, Brian Naberhuis, and others. We are also much indebted to discussions with a number of scientists elsewhere, including particularly Fritz Busse, Mike Cross, and Werner Pesch. This work was supported by the Department of Energy through Grant DE-FG03-87ER13738. 


\section{REFERENCES}

[1] For a recent review, see for instance, M. C. Cross and P.C. Hohenberg, Rev. Mod. Phys. 65, 851 (1993).

[2] V. Croquette, Contemp. Phys. 30, 113 (1989).

[3] V. Croquette, Contemp. Phys. 30, 153 (1989).

[4] E. Bodenschatz, J. R. de Bruyn, G. Ahlers, and D. S. Cannell, Phys. Rev. Lett. 67, 3078 (1991).

[5] E. Bodenschatz, D. S. Cannell, J. R. de Bruyn, R. Ecke, Y. Hu, K. Lerman, and G. Ahlers, Physica D 61, 77 (1992).

[6] S. W. Morris, E. Bodenschatz, D. S. Cannell, and G. Ahlers, Phys. Rev. Lett. 71, 2026 (1993).

[7] M. Assenheimer and V. Steinberg, Phys. Rev. Lett. 70, 3888 (1993).

[8] M. Assenheimer and V. Steinberg, Nature 367, 345 (1994).

[9] Y. Hu, R. E. Ecke, and G. Ahlers, Phys. Rev. E 48, 4399 (1993).

[10] L. Ning, Y. Hu, R. E. Ecke, and G. Ahlers, Phys. Rev. Lett. 71, 2216 (1993).

[11] Y. Hu, R. E. Ecke, and G. Ahlers, Phys. Rev. Lett. 72, 2191 (1994).

[12] Y. Hu, R. E. Ecke, and G. Ahlers, Phys. Rev. Lett. 74, 391 (1995).

[13] Y. Hu, R. E. Ecke, and G. Ahlers, Phys. Rev. E 51, 3263 (1995).

[14] R. E. Ecke, Y. Hu, R. Mainieri, and G. Ahlers, Science 269, 1704 (1995).

[15] S. W. Morris, E. Bodenschatz, D. S. Cannell, and G. Ahlers, Physica D 97, 164 (1996).

[16] J. R. de Bruyn, E. Bodenschatz, S. W. Morris, S. Trainoff, Y. Hu, D. S. Cannell, and G. Ahlers, Rev. Sci. Instrum. 67, 2043 (1996).

[17] J. Liu and G. Ahlers, Phys. Rev. Lett. 77, 3126 (1996).

[18] B. Plapp and E. Bodenschatz, Phys. Script. 67, 111 (1996).

[19] J. Liu and G. Ahlers, Phys. Rev. E 55, 6950 (1997).

[20] K. M. S. Bajaj, D. Cannell, and G. Ahlers, Phys. Rev. E 55, 4869 (1997).

[21] R. Cakmur, D. Egolf, B. Plapp, and E. Bodenschatz, Phys. Rev. Lett. 79, 1853 (1997).

[22] A. Schluter, D. Lortz, and F. H. Busse, J. Fluid Mech. 23, 129 (1965).

[23] Y. Hu, R. E. Ecke, and G. Ahlers, Phys. Rev. Lett. 74, 5040 (1995).

[24] K. M. S. Bajaj, J. Liu, B. Naberhuis, and G. Ahlers, Phys. Rev. Lett. 00, 00 (1998).

[25] G. Küppers and D. Lortz, J. Fluid Mech. 35, 609 (1969).

[26] G. Küppers, Phys. Lett. 32A, 7 (1970).

[27] R. M. Clever and F. H. Busse, J. Fluid Mech. 94, 609 (1979).

[28] F. H. Busse and K. E. Heikes, Science 208, 173 (1980).

[29] K. E. Heikes and F. H. Busse, Ann. N.Y. Acad. Sci. 357, 28 (1980).

[30] Y. Hu, R. E. Ecke, and G. Ahlers, Phys. Rev. E 55, 6928 (1997).

[31] Y. Hu, W. Pesch, G. Ahlers, and R. E. Ecke, Phys. Rev. E 00, 00 (1998).

[32] One exception is liquid helium. As the superfluid-transition temperature $2.176 \mathrm{~K}$ is approached from above, $\sigma$ vanishes. However, experiments are difficult because $\sigma$ varies from a value of order one to zero over a narrow temperature range of a few $\mathrm{mK}$, and because of the problem of flow visualization, which has only recently 
been achieved under the required cryogenic conditions (P. Lucas, A. Woodcraft, R. Matley, and W. Wong, International Workshop on Ultra-High Reynolds-Number Flows, Brookhaven National Laboratory, June 18 to 20, 1996). Other exceptions are liquid metals which have $\sigma=\mathcal{O}\left(10^{-2}\right)$ because of the large electronic contribution to the conductivity. However, it is not possible to explore the range $10^{-2} \lesssim \sigma \lesssim 0.7$ with them. Since liquid metals are not transparent to visible light, flow visualization is also a problem.

[33] T. Clune and E. Knobloch, Phys. Rev. E 47, 2536 (1993).

[34] Early theoretical evidence for the existence of a subcritical and tricritical bifurcation is contained in the work of Clever and Busse (CB)(Ref. [27]). More recent calculations of the tricritical line by Clune and Knobloch (Ref. [33]) are inconsistent with the result of CB. Using programs developed by W. Pesch, we re-calculated the tricritical line and obtained the result shown in Fig. 3 which is more detailed than, but agrees with that of $\mathrm{CB}$.

[35] K. Buhler and H. Oertel, J. Fluid Mech. 114, 261 (1982).

[36] J. J. Niemela and R. J. Donnelly, Phys. Rev. Lett. 57, 2524 (1986).

[37] F. Zhong, R. Ecke, and V. Steinberg, Physica D 51, 596 (1991).

[38] F. Zhong and R. Ecke, Chaos 2, 163 (1992).

[39] Y. Tu and M. Cross, Phys. Rev. Lett. 69, 2515 (1992).

[40] M. Fantz, R. Friedrich, M. Bestehorn, and H. Haken, Physica D 61, 147 (1992).

[41] M. Neufeld, R. Friedrich, and H. Haken, Z. Phys. B. 92, 243 (1993).

[42] M. Cross, D. Meiron, and Y. Tu, Chaos 4, 607 (1994).

[43] Y. Ponty, T. Passot, and P. Sulem, Phys. Rev. Lett. 00, 00 (1997).

[44] Recently it was shown in Ref. [31] that the data for $\xi$ and $\omega_{a}$ can be fit with a powerlaw and the expected theoretical leading exponents if large correction terms are allowed in the analysis.

[45] S. Chandrasekhar, Hydrodynamic and Hydromagnetic Stability (Oxford University Press, Oxford, 1961).

[46] M. E. Fisher, Phys. Rev. 176, 257 (1968). 